\title{
Rainbow Connection Number and the Diameter of Interval Graphs
}

\author{
Dr. A. Sudhakaraiah*, E.Gnana Deepika ${ }^{1}$, V. Rama Latha ${ }^{2}$ \\ (Department of Mathematics, S. V. University, Tirupati-517502, Andhra Pradesh, India.)
}

\begin{abstract}
When the numerical value of the labels is unimportant, we call them colors to indicate that they may be elements of any set. In graph theory, a connected component of an undirected graph is a subgraph in which any two vertices are connected to each other by paths. The rainbow connection number of a connected graph is the minimum number of colors needed to color its edges, so that every pair of its vertices is connected by at least one path in which no two edges are colored the same. In this paper we show that the rainbow connection number of an interval graph, which are of the form the rainbow connection number is less than or equal to the diameter of the graph $G$ plus one.
\end{abstract}

Keywords - diameter, eccentricity, interval graph, rainbow connection number, rainbow path.

\section{INTRODUCTION}

A Graph $G=(V, E)$ is an interval graph, if the vertex set $V$ can be put into one-to-one correspondence with a set of intervals $I$ on the real line $R$, such that two vertices are adjacent in $G$ if and only if their corresponding intervals have non-empty intersection. The set $I$ is called an interval representation of $G$ and $G$ is referred to as the intersection graph $I$. The intervals $I=\left\{i_{1}, i_{2}, \ldots ., i_{n}\right\}$ be an interval family, where each $i_{i}$ is an interval on the real line and $i_{i}=\left[a_{i}, b_{i}\right]$ for $i=1,2, \ldots ., n$, here $a_{i}$ is called left end point labeling and $b_{i}$ is the right end point labeling of $i_{i}$. Without loss of generality we assume that all end points of the interval in $I$ are distinct numbers between 1 and $2 n$. Two intervals $i$ and $j$ are said to be intersect each other if they have non-empty intersection. An edge color of a graph is a function from its edge set to the set of natural numbers. A path in an edge colored graph with no two edges sharing the same color is called a Rainbow Path. An edge colored graph is said to be rainbow connected if every pair of vertices is connected by at least one rainbow path, such a color is called a rainbow color of the graph. The minimum number of colors required to a connected graph is called its rainbow connection number [1,2,5], denoted by $r c(G)$. In graph theory, the rainbow connection number of a complete graph is one, that of a path is its length, that of an even cycle is its diameter[3]. The diameter of a graph $G$ is the maximum of eccentricity of all its vertices and is denoted by $\operatorname{diam}(G)$, that is $\operatorname{diam}(G)=\max \{\operatorname{ecc}(v): v \in V(G)\}$, where the maximum distance from a vertex $u$ to any vertex of $G$ is called eccentricity of the vertex $v$ and is denoted by ecc $(v)$, that is $\operatorname{ecc}(v)=\max \{d(u, v): u \in V(G)\}$, where as the distance between two vertices $u$ and $v$ of a graph is the length of the shortest path between them and is denoted by $d_{G}(u, v)$ or $d(u, v)$. To the rainbow colored graph it is enough to ensure that every edge of some spanning tree in the graph gets a distinct color. Hence it is an interval graph with minimum degree $\delta(G)$ [4] is two and the diameter "diam $(G)$ ".

\section{MAIN THEOREMS}

2.1 THEOREM : Let $I=\left\{i_{1}, i_{2}, \ldots . ., i_{n}\right\}$ be an interval family and $G$ is an interval graph with $\delta(G) \geq 2$ corresponding to $I$ such that $j \neq 1$ and $j$ is contained in $i$ and if there is at least one interval to the left of $j$ that intersect $j$, and at least one interval $k \neq i$, to the right of $j$ that intersect $j$ and similarly $G$ be a connected graph with $\delta(G) \geq 2$ then $\operatorname{rc}(G) \leq \operatorname{diam}(G)+1$.

PROOF : Let $I=\left\{i_{1}, i_{2}, \ldots . ., i_{n}\right\}$ be an interval family and $G$ is an interval graph corresponding to $I$. Suppose there is at least one interval $k \neq i$ to the right of $j$ that intersect $j$ then it is obvious that $j$ is www.iosrjournals.org 36|Page 
adjacent to $k$ that is $j$ is connected $k$, so that there will not be any disconnection in $G$. Since there is at least one interval to the left of $j$ that intersect $j$, there will not be any disconnection in $G$. Thus we get connected graph in $G$. Moreover there exists interval graphs threshold graphs and chain graphs with minimum degree at least two and rainbow connection number $\operatorname{rc}(G)$ equal to the corresponding upper bound above. There exists an interval graph $G$ with minimum degree at least two and $r c(G)<\operatorname{diam}(G)+1$ or $r c(G)=\operatorname{diam}(G)+1$. First we will prove that the following properties of $G$ which are distance, eccentricity, diameter. From $G$ the distance between two vertices $v_{1}$ and $v_{2}$ of a graph $G$ is the length of the shortest path between them and is denoted by $d_{G}\left(v_{1}, v_{2}\right)$. Next eccentricity is the maximum distance from a vertex $v_{1}$ to any vertex $v_{2}$ of $G$ and is denoted by $\operatorname{ecc}\left(v_{2}\right)$ that is $\operatorname{ecc}\left(v_{2}\right)=\max \left\{d_{G}\left(v_{1}, v_{2}\right): v_{1} \in V(G)\right\}$, also the diameter of $G$ is the maximum of eccentricity of all its vertices is denoted by $\operatorname{diam}(G)$ that is $\operatorname{diam}(G)=\max \left\{e\left(v_{2}\right): v_{2} \in V(G)\right\}$. Also we know that the assignment of colors to the edges of a graph $G$ corresponding to an interval family $I$, so that no two adjacent edges receiving the same color of $G$ as follows the rainbow connection number of $G$. Next we will prove that $r c(G) \leq \operatorname{diam}(G)+1$.

This procedure as follows with an illustration,

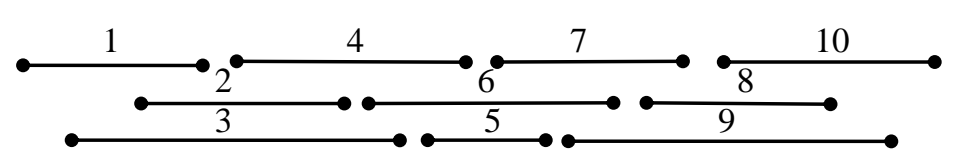

Fig.1: Interval Family $I$

An Interval graph as follows from an Interval family $I$ as in Fig.1, since an Interval graph indicated by the neighbourhood sets.

$\operatorname{nbd}[1]=\{1,2,3\}, \quad \operatorname{nbd}[2]=\{1,2,3,4\}$, $\operatorname{nbd}[5]=\{4,5,6,7\}, \quad \operatorname{nbd}[6]=\{3,4,5,6,7,9\}$, $[9]=\{6,7,8,9,10\}, \operatorname{nbd}[10]=\{8,9,10\}$ Intervalgraph $\mathrm{G}$ corresponding to an Interval family $\mathrm{I}$. Let us assume that $\left\{\mathrm{v}_{1}, \mathrm{v}_{2}, \ldots \ldots, \mathrm{v}_{10}\right\}$ Since

$\mathrm{C}\left(\mathrm{v}_{1}, \mathrm{v}_{2}\right)=1, \mathrm{C}\left(\mathrm{v}_{1}, \mathrm{v}_{3}\right)=2, \mathrm{C}\left(\mathrm{v}_{2}, \mathrm{v}_{3}\right)=3, \mathrm{C}\left(\mathrm{v}_{2}, \mathrm{v}_{4}\right)=2, \mathrm{C}\left(\mathrm{v}_{3}, \mathrm{v}_{4}\right)=1, \mathrm{C}\left(\mathrm{v}_{3}, \mathrm{v}_{6}\right)=4, \mathrm{C}\left(\mathrm{v}_{4}, \mathrm{v}_{5}\right)=4, \mathrm{C}\left(\mathrm{v}_{4}, \mathrm{v}_{6}\right)=3$, $\mathrm{C}\left(\mathrm{v}_{5}, \mathrm{v}_{6}\right)=1, \mathrm{C}\left(\mathrm{v}_{5}, \mathrm{v}_{7}\right)=2, \mathrm{C}\left(\mathrm{v}_{6}, \mathrm{v}_{7}\right)=5, \mathrm{C}\left(\mathrm{v}_{6}, \mathrm{v}_{9}\right)=2, \mathrm{C}\left(\mathrm{v}_{7}, \mathrm{v}_{8}\right)=2, \mathrm{C}\left(\mathrm{v}_{7}, \mathrm{v}_{9}\right)=1, \mathrm{C}\left(\mathrm{v}_{8}, \mathrm{v}_{9}\right)=3, \mathrm{C}\left(\mathrm{v}_{8}, \mathrm{v}_{10}\right)=1$, $\mathrm{C}\left(\mathrm{v}_{9}, \mathrm{v}_{10}\right)=5$

Therefore the set of colors are

$$
\begin{gathered}
\mathrm{C}=\{1,2,3,2,1,4,4,3,1,2,5,2,2,1,3,1,5\} \\
=\{1,2,3,4,5\} \\
\therefore r c(G)=5
\end{gathered}
$$

The distances of the path lengths from Interval graph $G$ as follows, $\operatorname{nbd}[3]=\{1,2,3,4,6\}, \operatorname{nbd}[4]=\{2,3,4,5,6\}$, $\operatorname{nbd}[7]=\{5,6,7,8,9\}, \operatorname{nbd}[8]=\{7,8,9,10\}, \operatorname{nbd}$

The rainbow edge colors as follows from an $I=\{1,2, \ldots ., 10\}=$

(1)




\begin{tabular}{|c|c|c|c|c|}
\hline $\mathrm{d}\left(\mathrm{v}_{1}, \mathrm{v}_{1}\right)$ & $\mathrm{d}\left(\mathrm{v}_{2}, \mathrm{v}_{1}\right)=1$ & $d\left(v_{3}, v_{1}\right)=1$ & $\mathrm{~d}\left(\mathrm{v}_{4}, \mathrm{v}_{1}\right)=2$ & $\mathrm{~d}\left(\mathrm{v}_{5}, \mathrm{v}_{1}\right)=3$ \\
\hline$=0$ & $\mathrm{~d}\left(\mathrm{v}_{2}, \mathrm{v}_{2}\right)=0$ & $d\left(v_{3}, v_{2}\right)=1$ & $\mathrm{~d}\left(\mathrm{v}_{4}, \mathrm{v}_{2}\right)=1$ & $\mathrm{~d}\left(\mathrm{v}_{5}, \mathrm{v}_{2}\right)=2$ \\
\hline$d\left(v_{1}, v_{2}\right)=1$ & $\mathrm{~d}\left(\mathrm{v}_{2}, \mathrm{v}_{3}\right)=1$ & $d\left(v_{3}, v_{3}\right)=0$ & $\mathrm{~d}\left(\mathrm{v}_{4}, \mathrm{v}_{3}\right)=1$ & $\mathrm{~d}\left(\mathrm{v}_{5}, \mathrm{v}_{3}\right)=2$ \\
\hline $\mathrm{d}\left(\mathrm{v}_{1}, \mathrm{v}_{3}\right)=1$ & $\mathrm{~d}\left(\mathrm{v}_{2}, \mathrm{v}_{4}\right)=1$ & $\mathrm{~d}\left(\mathrm{v}_{3}, \mathrm{v}_{4}\right)=1$ & $\mathrm{~d}\left(\mathrm{v}_{4}, \mathrm{v}_{4}\right)=0$ & $\mathrm{~d}\left(\mathrm{v}_{5}, \mathrm{v}_{4}\right)=1$ \\
\hline $\mathrm{d}\left(\mathrm{v}_{1}, \mathrm{v}_{4}\right)=2$ & $\mathrm{~d}\left(\mathrm{v}_{2}, \mathrm{v}_{5}\right)=2$ & $\mathrm{l}\left(\mathrm{v}_{3}, \mathrm{v}_{5}\right)=2$ & $\mathrm{~d}\left(\mathrm{v}_{3}, \mathrm{v}_{5}\right)=1$ & $\mathrm{~d}\left(\mathrm{v}_{5}, \mathrm{v}_{5}\right)=0$ \\
\hline$d\left(v_{1}, v_{5}\right)=3$ & $\left(\mathrm{v}_{2}, \mathrm{v}_{6}\right)=2$ & $1\left(v_{3}, v_{6}\right)=1$ & $d\left(v_{4}, v_{6}\right)=1$ & $\mathrm{~d}\left(\mathrm{v}_{5}, \mathrm{v}_{6}\right)=1$ \\
\hline$(6)=2$ & $=3$ & $=2$ & $\left(v_{4}, v_{7}\right)=2$ & $\mathrm{~d}\left(\mathrm{v}_{5}, \mathrm{v}_{7}\right)=1$ \\
\hline $\mathrm{d}\left(\mathrm{v}_{1}, \mathrm{v}_{7}\right)=3$ & $=4$ & $=3$ & $\left(v_{4}, v_{8}\right)=3$ & $\mathrm{~d}\left(\mathrm{v}_{5}, \mathrm{v}_{8}\right)=2$ \\
\hline $\mathrm{d}\left(\mathrm{v}_{1}, \mathrm{v}_{8}\right)=4$ &,$\left.v_{9}\right)=3$ & $\left.v_{9}\right)=2$ & $\mathrm{~d}\left(\mathrm{v}_{4}, \mathrm{v}_{9}\right)=2$ & $\mathrm{~d}\left(\mathrm{v}_{5}, \mathrm{v}_{9}\right)=2$ \\
\hline$d\left(v_{1}, v_{9}\right)=3$ & $\mathrm{~d}\left(\mathrm{v}_{2}, \mathrm{v}_{10}\right)=4$ & $\mathrm{~d}\left(\mathrm{v}_{3}, \mathrm{v}_{10}\right)=3$ & $\mathrm{~d}\left(\mathrm{v}_{4}, \mathrm{v}_{10}\right)=3$ & $\mathrm{~d}\left(\mathrm{v}_{5}, \mathrm{v}_{10}\right)=3$ \\
\hline $\mathrm{d}\left(\mathrm{v}_{1}, \mathrm{v}_{10}\right)=4$ & & & & \\
\hline $\mathrm{d}\left(\mathrm{v}_{6}, \mathrm{v}_{1}\right)=2$ & $\mathrm{~d}\left(\mathrm{v}_{7}, \mathrm{v}_{1}\right)=3$ & $\mathrm{~d}\left(\mathrm{v}_{8}, \mathrm{v}_{1}\right)=4$ & $\mathrm{~d}\left(\mathrm{v}_{9}, \mathrm{v}_{1}\right)=3$ & $\mathrm{~d}\left(\mathrm{v}_{10}, \mathrm{v}_{1}\right)=4$ \\
\hline $\mathrm{d}\left(\mathrm{v}_{6}, \mathrm{v}_{2}\right)=2$ & $d\left(v_{7}, v_{2}\right)=3$ & $\mathrm{~d}\left(\mathrm{v}_{8}, \mathrm{v}_{2}\right)=4$ & $\mathrm{~d}\left(\mathrm{v}_{9}, \mathrm{v}_{2}\right)=3$ & $\mathrm{~d}\left(\mathrm{v}_{10}, \mathrm{v}_{2}\right)=4$ \\
\hline $\mathrm{d}\left(\mathrm{v}_{6}, \mathrm{v}_{3}\right)=1$ & $d\left(v_{7}, v_{3}\right)=2$ & $\mathrm{~d}\left(\mathrm{v}_{8}, \mathrm{v}_{3}\right)=3$ & $\mathrm{~d}\left(\mathrm{v}_{9}, \mathrm{v}_{3}\right)=2$ & $\mathrm{~d}\left(\mathrm{v}_{10}, \mathrm{v}_{3}\right)=3$ \\
\hline $\mathrm{d}\left(\mathrm{v}_{6}, \mathrm{v}_{4}\right)=1$ & $\mathrm{~d}\left(\mathrm{v}_{7}, \mathrm{v}_{4}\right)=2$ & $d\left(v_{8}, v_{4}\right)=3$ & $\mathrm{~d}\left(\mathrm{v}_{9}, \mathrm{v}_{4}\right)=2$ & $\mathrm{~d}\left(\mathrm{v}_{10}, \mathrm{v}_{4}\right)=3$ \\
\hline $\mathrm{d}\left(\mathrm{v}_{6}, \mathrm{v}_{5}\right)=1$ & $\mathrm{~d}\left(\mathrm{v}_{7}, \mathrm{v}_{5}\right)=1$ & $\mathrm{~d}\left(\mathrm{v}_{8}, \mathrm{v}_{5}\right)=2$ & $\mathrm{~d}\left(\mathrm{v}_{9}, \mathrm{v}_{5}\right)=2$ & $\mathrm{~d}\left(\mathrm{v}_{10}, \mathrm{v}_{5}\right)=3$ \\
\hline $\mathrm{d}\left(\mathrm{v}_{6}, \mathrm{v}_{6}\right)=0$ & $\mathrm{~d}\left(\mathrm{v}_{7}, \mathrm{v}_{6}\right)=1$ & $d\left(v_{8}, v_{6}\right)=2$ & $\mathrm{~d}\left(\mathrm{v}_{9}, \mathrm{v}_{6}\right)=1$ & $\mathrm{~d}\left(\mathrm{v}_{10}, \mathrm{v}_{6}\right)=2$ \\
\hline $\mathrm{d}\left(\mathrm{v}_{6}, \mathrm{v}_{7}\right)=1$ & $\mathrm{~d}\left(\mathrm{v}_{7}, \mathrm{v}_{7}\right)=0$ & $d\left(v_{8}, v_{7}\right)=1$ & $\mathrm{~d}\left(\mathrm{v}_{9}, \mathrm{v}_{7}\right)=1$ & $\mathrm{~d}\left(\mathrm{v}_{10}, \mathrm{v}_{7}\right)=2$ \\
\hline $\mathrm{d}\left(\mathrm{v}_{6}, \mathrm{v}_{8}\right)=2$ & $\mathrm{~d}\left(\mathrm{v}_{7}, \mathrm{v}_{8}\right)=1$ & $\mathrm{~d}\left(\mathrm{v}_{8}, \mathrm{v}_{8}\right)=0$ & $\mathrm{~d}\left(\mathrm{v}_{9}, \mathrm{v}_{8}\right)=1$ & $\mathrm{~d}\left(\mathrm{v}_{10}, \mathrm{v}_{8}\right)=1$ \\
\hline$d\left(v_{6}, v_{9}\right)=1$ & $\left(v_{7}, v_{9}\right)=1$ & $\mathrm{~d}\left(\mathrm{v}_{8}, \mathrm{v}_{9}\right)=1$ & $\mathrm{~d}\left(\mathrm{v}_{9}, \mathrm{v}_{9}\right)=0$ & $\mathrm{~d}\left(\mathrm{v}_{10}, \mathrm{v}_{9}\right)=1$ \\
\hline $\mathrm{d}\left(\mathrm{v}_{6}, \mathrm{v}_{10}\right)=2$ & $\mathrm{~d}\left(\mathrm{v}_{7}, \mathrm{v}_{10}\right)=2$ & $\mathrm{~d}\left(\mathrm{v}_{8}, \mathrm{v}_{10}\right)=1$ & $\mathrm{~d}\left(\mathrm{v}_{9}, \mathrm{v}_{10}\right)=1$ & $\mathrm{~d}\left(\mathrm{v}_{10}, \mathrm{v}_{10}\right)=0$ \\
\hline
\end{tabular}

The eccentricity of the vertices is as follows,

$$
\operatorname{ecc}(v)=\max _{x \in V(G)} d(v, x)
$$

$\operatorname{ecc}\left(\mathrm{v}_{1}\right)=\max \left\{\mathrm{d}\left(\mathrm{v}_{1}, \mathrm{v}_{1}\right), \mathrm{d}\left(\mathrm{v}_{1}, \mathrm{v}_{2}\right), \mathrm{d}\left(\mathrm{v}_{1}, \mathrm{v}_{3}\right), \mathrm{d}\left(\mathrm{v}_{1}, \mathrm{v}_{4}\right), \quad \mathrm{d}\left(\mathrm{v}_{1}, \mathrm{v}_{5}\right), \mathrm{d}\left(\mathrm{v}_{1}, \mathrm{v}_{6}\right), \mathrm{d}\left(\mathrm{v}_{1}, \mathrm{v}_{7}\right), \mathrm{d}\left(\mathrm{v}_{1}, \mathrm{v}_{8}\right), \mathrm{d}\left(\mathrm{v}_{1}, \mathrm{v}_{9}\right), \mathrm{d}\left(\mathrm{v}_{1}, \mathrm{v}_{10}\right)\right\}$ $=\max \{0,1,1,2,3,2,3,4,3,4\}=4$

$\operatorname{ecc}\left(\mathrm{v}_{2}\right)=\max \left\{\mathrm{d}\left(\mathrm{v}_{2}, \mathrm{v}_{1}\right), \mathrm{d}\left(\mathrm{v}_{2}, \mathrm{v}_{2}\right), \mathrm{d}\left(\mathrm{v}_{2}, \mathrm{v}_{3}\right), \mathrm{d}\left(\mathrm{v}_{2}, \mathrm{v}_{4}\right), \mathrm{d}\left(\mathrm{v}_{2}, \mathrm{v}_{5}\right), \mathrm{d}\left(\mathrm{v}_{2}, \mathrm{v}_{6}\right), \mathrm{d}\left(\mathrm{v}_{2}, \mathrm{v}_{7}\right), \mathrm{d}\left(\mathrm{v}_{2}, \mathrm{v}_{8}\right), \mathrm{d}\left(\mathrm{v}_{2}, \mathrm{v}_{9}\right), \mathrm{d}\left(\mathrm{v}_{2}, \mathrm{v}_{10}\right)\right\}$ $=\max \{1,0,1,1,2,2,3,4,3,4\}=4$

$\operatorname{ecc}\left(\mathrm{v}_{3}\right)=\max \left\{\mathrm{d}\left(\mathrm{v}_{3}, \mathrm{v}_{1}\right), \mathrm{d}\left(\mathrm{v}_{3}, \mathrm{v}_{2}\right), \mathrm{d}\left(\mathrm{v}_{3}, \mathrm{v}_{3}\right), \mathrm{d}\left(\mathrm{v}_{3}, \mathrm{v}_{4}\right), \mathrm{d}\left(\mathrm{v}_{3}, \mathrm{v}_{5}\right), \mathrm{d}\left(\mathrm{v}_{3}, \mathrm{v}_{6}\right), \mathrm{d}\left(\mathrm{v}_{3}, \mathrm{v}_{7}\right), \mathrm{d}\left(\mathrm{v}_{3}, \mathrm{v}_{8}\right), \mathrm{d}\left(\mathrm{v}_{3}, \mathrm{v}_{9}\right), \mathrm{d}\left(\mathrm{v}_{3}, \mathrm{v}_{10}\right)\right\}$ $=\max \{1,1,0,1,2,1,2,3,2,3\}=3$

$\operatorname{ecc}\left(\mathrm{v}_{4}\right)=\max \left\{\mathrm{d}\left(\mathrm{v}_{4}, \mathrm{v}_{1}\right), \mathrm{d}\left(\mathrm{v}_{4}, \mathrm{v}_{2}\right), \mathrm{d}\left(\mathrm{v}_{4}, \mathrm{v}_{3}\right), \mathrm{d}\left(\mathrm{v}_{4}, \mathrm{v}_{4}\right), \mathrm{d}\left(\mathrm{v}_{4}, \mathrm{v}_{5}\right), \mathrm{d}\left(\mathrm{v}_{4}, \mathrm{v}_{6}\right), \mathrm{d}\left(\mathrm{v}_{4}, \mathrm{v}_{7}\right), \mathrm{d}\left(\mathrm{v}_{4}, \mathrm{v}_{8}\right), \mathrm{d}\left(\mathrm{v}_{4}, \mathrm{v}_{9}\right), \mathrm{d}\left(\mathrm{v}_{4}, \mathrm{v}_{10}\right)\right\}$ $=\max \{2,1,1,0,1,1,2,3,2,3\}=3$

$\operatorname{ecc}\left(\mathrm{v}_{5}\right)=\max \left\{\mathrm{d}\left(\mathrm{v}_{5}, \mathrm{v}_{1}\right), \mathrm{d}\left(\mathrm{v}_{5}, \mathrm{v}_{2}\right), \mathrm{d}\left(\mathrm{v}_{5}, \mathrm{v}_{3}\right), \mathrm{d}\left(\mathrm{v}_{5}, \mathrm{v}_{4}\right), \mathrm{d}\left(\mathrm{v}_{5}, \mathrm{v}_{5}\right), \mathrm{d}\left(\mathrm{v}_{5}, \mathrm{v}_{6}\right), \mathrm{d}\left(\mathrm{v}_{5}, \mathrm{v}_{7}\right), \mathrm{d}\left(\mathrm{v}_{5}, \mathrm{v}_{8}\right), \mathrm{d}\left(\mathrm{v}_{5}, \mathrm{v}_{9}\right), \mathrm{d}\left(\mathrm{v}_{5}, \mathrm{v}_{10}\right)\right\}$ $=\max \{3,2,2,1,0,1,1,2,2,3\}=3$

$\operatorname{ecc}\left(\mathrm{v}_{6}\right)=\max \left\{\mathrm{d}\left(\mathrm{v}_{6}, \mathrm{v}_{1}\right), \mathrm{d}\left(\mathrm{v}_{6}, \mathrm{v}_{2}\right), \mathrm{d}\left(\mathrm{v}_{6}, \mathrm{v}_{3}\right), \mathrm{d}\left(\mathrm{v}_{6}, \mathrm{v}_{4}\right), \mathrm{d}\left(\mathrm{v}_{6}, \mathrm{v}_{5}\right), \mathrm{d}\left(\mathrm{v}_{6}, \mathrm{v}_{6}\right), \mathrm{d}\left(\mathrm{v}_{6}, \mathrm{v}_{7}\right), \mathrm{d}\left(\mathrm{v}_{6}, \mathrm{v}_{8}\right), \mathrm{d}\left(\mathrm{v}_{6}, \mathrm{v}_{9}\right), \mathrm{d}\left(\mathrm{v}_{6}, \mathrm{v}_{10}\right)\right\}$ $=\max \{2,2,1,1,1,0,1,2,1,2\}=2$

$\operatorname{ecc}\left(\mathrm{v}_{7}\right)=\max \left\{\mathrm{d}\left(\mathrm{v}_{7}, \mathrm{v}_{1}\right), \mathrm{d}\left(\mathrm{v}_{7}, \mathrm{v}_{2}\right), \mathrm{d}\left(\mathrm{v}_{7}, \mathrm{v}_{3}\right), \mathrm{d}\left(\mathrm{v}_{7}, \mathrm{v}_{4}\right), \mathrm{d}\left(\mathrm{v}_{7}, \mathrm{v}_{5}\right), \mathrm{d}\left(\mathrm{v}_{7}, \mathrm{v}_{6}\right), \mathrm{d}\left(\mathrm{v}_{7}, \mathrm{v}_{7}\right), \mathrm{d}\left(\mathrm{v}_{7}, \mathrm{v}_{8}\right), \mathrm{d}\left(\mathrm{v}_{7}, \mathrm{v}_{9}\right), \mathrm{d}\left(\mathrm{v}_{7}, \mathrm{v}_{10}\right)\right\}$ $=\max \{3,3,2,2,1,1,0,1,1,2\}=3$

$\operatorname{ecc}\left(\mathrm{v}_{8}\right)=\max \left\{\mathrm{d}\left(\mathrm{v}_{8}, \mathrm{v}_{1}\right), \mathrm{d}\left(\mathrm{v}_{8}, \mathrm{v}_{2}\right), \mathrm{d}\left(\mathrm{v}_{8}, \mathrm{v}_{3}\right), \mathrm{d}\left(\mathrm{v}_{8}, \mathrm{v}_{4}\right), \mathrm{d}\left(\mathrm{v}_{8}, \mathrm{v}_{5}\right), \mathrm{d}\left(\mathrm{v}_{8}, \mathrm{v}_{6}\right), \mathrm{d}\left(\mathrm{v}_{8}, \mathrm{v}_{7}\right), \mathrm{d}\left(\mathrm{v}_{8}, \mathrm{v}_{8}\right), \mathrm{d}\left(\mathrm{v}_{8}, \mathrm{v}_{9}\right), \mathrm{d}\left(\mathrm{v}_{8}, \mathrm{v}_{10}\right)\right\}$ $=\max \{4,4,3,3,2,2,1,0,1,1\}=4$

$\operatorname{ecc}\left(\mathrm{v}_{9}\right)=\max \left\{\mathrm{d}\left(\mathrm{v}_{9}, \mathrm{v}_{1}\right), \mathrm{d}\left(\mathrm{v}_{9}, \mathrm{v}_{2}\right), \mathrm{d}\left(\mathrm{v}_{9}, \mathrm{v}_{3}\right), \mathrm{d}\left(\mathrm{v}_{9}, \mathrm{v}_{4}\right), \mathrm{d}\left(\mathrm{v}_{9}, \mathrm{v}_{5}\right), \mathrm{d}\left(\mathrm{v}_{9}, \mathrm{v}_{6}\right), \mathrm{d}\left(\mathrm{v}_{9}, \mathrm{v}_{7}\right), \mathrm{d}\left(\mathrm{v}_{9}, \mathrm{v}_{8}\right), \mathrm{d}\left(\mathrm{v}_{9}, \mathrm{v}_{9}\right), \mathrm{d}\left(\mathrm{v}_{9}, \mathrm{v}_{10}\right)\right\}$ $=\max \{3,3,2,2,2,1,1,1,0,1\}=3$

$\operatorname{ecc}\left(\mathrm{v}_{10}\right)=\max \left\{d\left(\mathrm{v}_{10}, \mathrm{v}_{1}\right), \mathrm{d}\left(\mathrm{v}_{10}, \mathrm{v}_{2}\right), \mathrm{d}\left(\mathrm{v}_{10}, \mathrm{v}_{3}\right), \mathrm{d}\left(\mathrm{v}_{10}, \mathrm{v}_{4}\right), \mathrm{d}\left(\mathrm{v}_{10}, \mathrm{v}_{5}\right), \mathrm{d}\left(\mathrm{v}_{10}, \mathrm{v}_{6}\right), \mathrm{d}\left(\mathrm{v}_{10}, \mathrm{v}_{7}\right), \mathrm{d}\left(\mathrm{v}_{10}, \mathrm{v}_{8}\right), \mathrm{d}\left(\mathrm{v}_{10}, \mathrm{v}_{9}\right)\right.$, $\left.\mathrm{d}\left(\mathrm{v}_{10}, \mathrm{v}_{10}\right)\right\}$ $=\max \{4,4,3,3,3,2,2,1,1,0\}=4$

The diameter of a graph $G$ is the maximum of eccentricity of all its vertices and is denoted by $\operatorname{diam}(G)$. That is $\operatorname{diam}(G)=\max \{e(v): v \in V(G)\}$

$$
\begin{aligned}
& \operatorname{diam}(G)= \max \left\{\operatorname{ecc}\left(v_{1}\right), \operatorname{ecc}\left(v_{2}\right), \operatorname{ecc}\left(v_{3}\right), \operatorname{ecc}\left(v_{4}\right), \operatorname{ecc}\left(v_{5}\right),\right. \\
&\left.\operatorname{ecc}\left(v_{6}\right), \operatorname{ecc}\left(v_{7}\right), \operatorname{ecc}\left(v_{8}\right), \operatorname{ecc}\left(v_{9}\right), \operatorname{ecc}\left(v_{10}\right)\right\} \\
&= \max \{4,4,3,3,3,2,3,4,3,4\}=4 \\
& \therefore \operatorname{rc}(\mathrm{G})=5, \operatorname{diam}(\mathrm{G})+1=5 \\
& \therefore \quad \operatorname{rc}(G) \leq \operatorname{diam}(G)+1
\end{aligned}
$$


2.2 THEOREM : Let $I=\left\{i_{1}, i_{2}, \ldots . ., i_{n}\right\}$ be an interval family and $G$ is an interval graph with $\delta(G) \geq 2$ corresponding to $I$. If $i$ and $j$ are two intervals in $I$ such that $i \neq 1, j=1$ and $j$ is contained in $i$ and if there is one or more intervals other than $i$ that intersect $j$, then the rainbow connection number $r c(G) \leq \operatorname{diam}(G)+1$.

PROOF : Suppose, $G$ is an interval graph corresponding to an interval family $I$. Let $I=\left\{i_{1}, i_{2}, \ldots \ldots, i_{n}\right\}$ be an interval family. In this we have $j=1$ be the interval contained in $i$, where $i \neq 1$, also we have $k$ is an interval, $k \neq i$ and $k$ intersect $j$. We must have $G$ is connected. The procedure as explained in theorem 1, next we will prove that $r c(G) \leq \operatorname{diam}(G)+1$ with the following illustration.

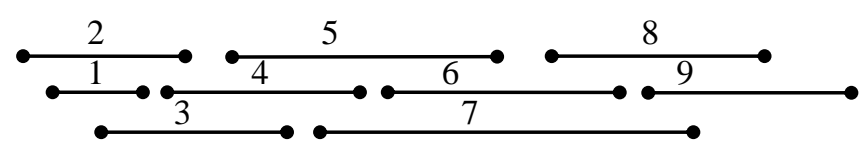

Fig.2: Interval family $I$

The Interval graph $G$ is given by the neighbourhood sets as follows from Interval family $I$ as in Fig.2 $\operatorname{nbd}[1]=\{1,2,3\}, \quad \operatorname{nbd}[2]=\{1,2,3,4\}$,

$\operatorname{nbd}[3]=\{1,2,3,4,5\}, \operatorname{nbd}[4]=\{2,3,4,5,7\}$,

$\operatorname{nbd}[5]=\{3,4,5,6,7\}, \quad \operatorname{nbd}[6]=\{5,6,7,8\}$,

$\operatorname{nbd}[7]=\{4,5,6,7,8,9\}, \operatorname{nbd}[8]=\{6,7,8,9\}$,

$\operatorname{nbd}[9]=\{7,8,9\}$

In this regard the rainbow edge colors of $G$ are,

$\mathrm{C}\left(\mathrm{v}_{1}, \mathrm{v}_{2}\right)=1, \mathrm{C}\left(\mathrm{v}_{1}, \mathrm{v}_{3}\right)=2, \mathrm{C}\left(\mathrm{v}_{2}, \mathrm{v}_{3}\right)=2, \mathrm{C}\left(\mathrm{v}_{2}, \mathrm{v}_{4}\right)=3$,

$\mathrm{C}\left(\mathrm{v}_{3}, \mathrm{v}_{4}\right)=1, \mathrm{C}\left(\mathrm{v}_{3}, \mathrm{v}_{5}\right)=4, \mathrm{C}\left(\mathrm{v}_{4}, \mathrm{v}_{5}\right)=2, \mathrm{C}\left(\mathrm{v}_{4}, \mathrm{v}_{7}\right)=4, \mathrm{C}\left(\mathrm{v}_{5}, \mathrm{v}_{6}\right)=1, \mathrm{C}\left(\mathrm{v}_{5}, \mathrm{v}_{7}\right)=3, \mathrm{C}\left(\mathrm{v}_{6}, \mathrm{v}_{7}\right)=2, \mathrm{C}\left(\mathrm{v}_{6}, \mathrm{v}_{8}\right)=3$,

$\mathrm{C}\left(\mathrm{v}_{7}, \mathrm{v}_{8}\right)=1, \mathrm{C}\left(\mathrm{v}_{7}, \mathrm{v}_{9}\right)=5, \mathrm{C}\left(\mathrm{v}_{8}, \mathrm{v}_{9}\right)=2$.

Therefore the set of colors are

$$
\begin{gathered}
\mathrm{C}=\{1,2,2,3,1,4,2,4,1,3,2,3,1,5,2\} \\
=\{1,2,3,4,5\} \\
\therefore r c(G)=5
\end{gathered}
$$

The distances of the path lengths from Interval graph $G$ as follows,

$$
\begin{aligned}
& \mathrm{d}\left(\mathrm{v}_{1}, \mathrm{v}_{1}\right)=0 \quad \mathrm{~d}\left(\mathrm{v}_{2}, \mathrm{v}_{1}\right)=1 \quad \mathrm{~d}\left(\mathrm{v}_{3}, \mathrm{v}_{1}\right)=1 \quad \mathrm{~d}\left(\mathrm{v}_{4}, \mathrm{v}_{1}\right)=2 \quad \mathrm{~d}\left(\mathrm{v}_{5}, \mathrm{v}_{1}\right)=2 \\
& \mathrm{~d}\left(\mathrm{v}_{1}, \mathrm{v}_{2}\right)=1 \quad \mathrm{~d}\left(\mathrm{v}_{2}, \mathrm{v}_{2}\right)=0 \quad \mathrm{~d}\left(\mathrm{v}_{3}, \mathrm{v}_{2}\right)=1 \quad \mathrm{~d}\left(\mathrm{v}_{4}, \mathrm{v}_{2}\right)=1 \quad \mathrm{~d}\left(\mathrm{v}_{5}, \mathrm{v}_{2}\right)=2 \\
& \mathrm{~d}\left(\mathrm{v}_{1}, \mathrm{v}_{3}\right)=1 \quad \mathrm{~d}\left(\mathrm{v}_{2}, \mathrm{v}_{3}\right)=1 \quad \mathrm{~d}\left(\mathrm{v}_{3}, \mathrm{v}_{3}\right)=0 \quad \mathrm{~d}\left(\mathrm{v}_{4}, \mathrm{v}_{3}\right)=1 \quad \mathrm{~d}\left(\mathrm{v}_{5}, \mathrm{v}_{3}\right)=1 \\
& \mathrm{~d}\left(\mathrm{v}_{1}, \mathrm{v}_{4}\right)=2 \quad \mathrm{~d}\left(\mathrm{v}_{2}, \mathrm{v}_{4}\right)=1 \quad \mathrm{~d}\left(\mathrm{v}_{3}, \mathrm{v}_{4}\right)=1 \quad \mathrm{~d}\left(\mathrm{v}_{4}, \mathrm{v}_{4}\right)=0 \quad \mathrm{~d}\left(\mathrm{v}_{5}, \mathrm{v}_{4}\right)=1 \\
& \begin{array}{lllll}
\mathrm{d}\left(\mathrm{v}_{1}, \mathrm{v}_{5}\right)=2 & \mathrm{~d}\left(\mathrm{v}_{2}, \mathrm{v}_{5}\right)=2 & \mathrm{~d}\left(\mathrm{v}_{3}, \mathrm{v}_{5}\right)=1 & \mathrm{~d}\left(\mathrm{v}_{4}, \mathrm{v}_{5}\right)=1 & \mathrm{~d}\left(\mathrm{v}_{5}, \mathrm{v}_{4}\right)=1 \\
\mathrm{~d}\left(\mathrm{v}_{5}, \mathrm{v}_{5}\right)=0
\end{array} \\
& \begin{array}{lllll}
\mathrm{d}\left(\mathrm{v}_{1}, \mathrm{v}_{6}\right)=3 & \mathrm{~d}\left(\mathrm{v}_{2}, \mathrm{v}_{6}\right)=3 & \mathrm{~d}\left(\mathrm{v}_{3}, \mathrm{v}_{6}\right)=2 & \mathrm{~d}\left(\mathrm{v}_{4}, \mathrm{v}_{6}\right)=2 & \mathrm{~d}\left(\mathrm{v}_{5}, \mathrm{v}_{5}\right)=0 \\
\mathrm{~d}\left(\mathrm{v}_{1}, \mathrm{v}_{7}\right)=3 & \mathrm{~d}\left(\mathrm{v}_{2}, \mathrm{v}_{7}\right)=2 & \mathrm{~d}\left(\mathrm{v}_{3}, \mathrm{v}_{7}\right)=2 & \mathrm{~d}\left(\mathrm{v}_{4}, \mathrm{v}_{7}\right)=1 & \mathrm{~d}\left(\mathrm{v}_{5}, \mathrm{v}_{6}\right)=1
\end{array} \\
& \mathrm{~d}\left(\mathrm{v}_{1}, \mathrm{v}_{8}\right)=4 \quad \mathrm{~d}\left(\mathrm{v}_{2}, \mathrm{v}_{8}\right)=3 \quad \mathrm{~d}\left(\mathrm{v}_{3}, \mathrm{v}_{8}\right)=3 \quad \mathrm{~d}\left(\mathrm{v}_{4}, \mathrm{v}_{8}\right)=2 \quad \mathrm{~d}\left(\mathrm{v}_{5}, \mathrm{v}_{7}\right)=1 \\
& \mathrm{~d}\left(\mathrm{v}_{1}, \mathrm{v}_{9}\right)=4 \quad \mathrm{~d}\left(\mathrm{v}_{2}, \mathrm{v}_{9}\right)=3 \quad \mathrm{~d}\left(\mathrm{v}_{3}, \mathrm{v}_{9}\right)=3 \quad \mathrm{~d}\left(\mathrm{v}_{4}, \mathrm{v}_{9}\right)=2 \quad \mathrm{~d}\left(\mathrm{v}_{5}, \mathrm{v}_{8}\right)=2 \\
& \mathrm{~d}\left(\mathrm{v}_{6}, \mathrm{v}_{1}\right)=3 \quad \mathrm{~d}\left(\mathrm{v}_{7}, \mathrm{v}_{1}\right)=3 \quad \mathrm{~d}\left(\mathrm{v}_{8}, \mathrm{v}_{1}\right)=4 \quad \mathrm{~d}\left(\mathrm{v}_{9}, \mathrm{v}_{1}\right)=4 \quad \mathrm{~d}\left(\mathrm{v}_{5}, \mathrm{v}_{9}\right)=2 \\
& \mathrm{~d}\left(\mathrm{v}_{6}, \mathrm{v}_{2}\right)=3 \quad \mathrm{~d}\left(\mathrm{v}_{7}, \mathrm{v}_{2}\right)=2 \quad \mathrm{~d}\left(\mathrm{v}_{8}, \mathrm{v}_{2}\right)=3 \quad \mathrm{~d}\left(\mathrm{v}_{9}, \mathrm{v}_{2}\right)=3 \\
& \mathrm{~d}\left(\mathrm{v}_{6}, \mathrm{v}_{3}\right)=2 \quad \mathrm{~d}\left(\mathrm{v}_{7}, \mathrm{v}_{3}\right)=2 \quad \mathrm{~d}\left(\mathrm{v}_{8}, \mathrm{v}_{3}\right)=3 \quad \mathrm{~d}\left(\mathrm{v}_{9}, \mathrm{v}_{3}\right)=3 \\
& \mathrm{~d}\left(\mathrm{v}_{6}, \mathrm{v}_{4}\right)=2 \quad \mathrm{~d}\left(\mathrm{v}_{7}, \mathrm{v}_{4}\right)=1 \quad \mathrm{~d}\left(\mathrm{v}_{8}, \mathrm{v}_{4}\right)=2 \quad \mathrm{~d}\left(\mathrm{v}_{9}, \mathrm{v}_{4}\right)=2 \\
& \mathrm{~d}\left(\mathrm{v}_{6}, \mathrm{v}_{5}\right)=1 \quad \mathrm{~d}\left(\mathrm{v}_{7}, \mathrm{v}_{5}\right)=1 \quad \mathrm{~d}\left(\mathrm{v}_{8}, \mathrm{v}_{5}\right)=2 \quad \mathrm{~d}\left(\mathrm{v}_{9}, \mathrm{v}_{5}\right)=2 \\
& \mathrm{~d}\left(\mathrm{v}_{6}, \mathrm{v}_{6}\right)=0 \quad \mathrm{~d}\left(\mathrm{v}_{7}, \mathrm{v}_{6}\right)=1 \quad \mathrm{~d}\left(\mathrm{v}_{8}, \mathrm{v}_{6}\right)=1 \quad \mathrm{~d}\left(\mathrm{v}_{9}, \mathrm{v}_{6}\right)=2 \\
& \mathrm{~d}\left(\mathrm{v}_{6}, \mathrm{v}_{7}\right)=1 \quad \mathrm{~d}\left(\mathrm{v}_{7}, \mathrm{v}_{7}\right)=0 \quad \mathrm{~d}\left(\mathrm{v}_{8}, \mathrm{v}_{7}\right)=1 \quad \mathrm{~d}\left(\mathrm{v}_{9}, \mathrm{v}_{7}\right)=1 \\
& \mathrm{~d}\left(\mathrm{v}_{6}, \mathrm{v}_{8}\right)=1 \quad \mathrm{~d}\left(\mathrm{v}_{7}, \mathrm{v}_{8}\right)=1 \quad \mathrm{~d}\left(\mathrm{v}_{8}, \mathrm{v}_{8}\right)=0 \quad \mathrm{~d}\left(\mathrm{v}_{9}, \mathrm{v}_{8}\right)=1 \\
& \mathrm{~d}\left(\mathrm{v}_{6}, \mathrm{v}_{9}\right)=2 \quad \mathrm{~d}\left(\mathrm{v}_{7}, \mathrm{v}_{9}\right)=1 \quad \mathrm{~d}\left(\mathrm{v}_{8}, \mathrm{v}_{9}\right)=1 \quad \mathrm{~d}\left(\mathrm{v}_{9}, \mathrm{v}_{9}\right)=0
\end{aligned}
$$

The eccentricity of the vertices is as follows, 


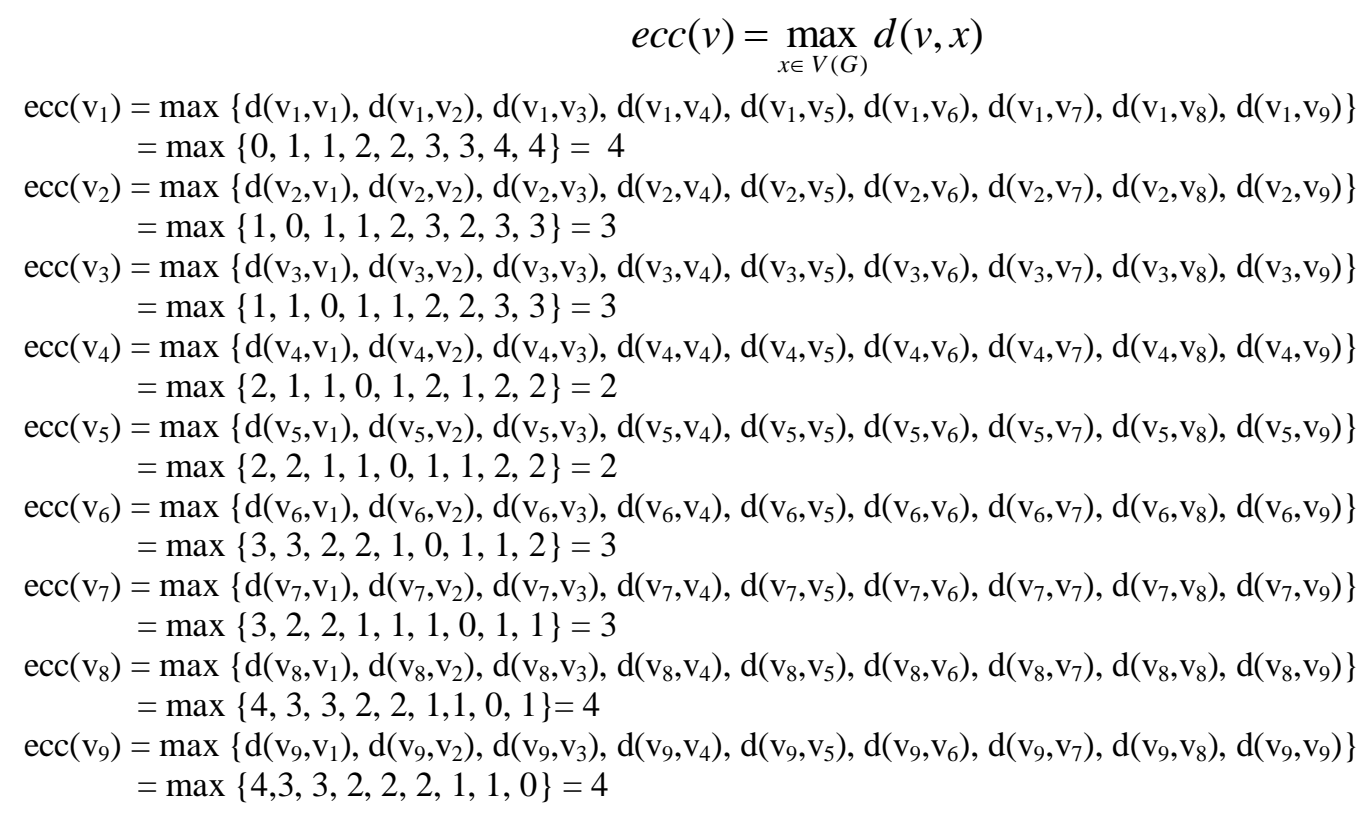

The diameter of a graph $G$ is the maximum of eccentricity of all its vertices and is denoted by $\operatorname{diam}(G)$

That is $\operatorname{diam}(G)=\max \{e(v): v \in V(G)\}$

$$
\begin{gathered}
\operatorname{diam}(G)=\max \left\{\operatorname{ecc}\left(v_{1}\right), \operatorname{ecc}\left(v_{2}\right), \operatorname{ecc}\left(v_{3}\right), \operatorname{ecc}\left(v_{4}\right),\right. \\
\left.\operatorname{ecc}\left(v_{5}\right), \operatorname{ecc}\left(v_{6}\right), \operatorname{ecc}\left(v_{7}\right), \operatorname{ecc}\left(v_{8}\right), \operatorname{ecc}\left(v_{9}\right)\right\} \\
=\max \{4,3,3,2,2,3,3,4,4\}=4 \\
\therefore \operatorname{rc}(\mathrm{G})=5, \operatorname{diam}(\mathrm{G})+1=5 \\
\therefore \operatorname{rc}(G) \leq \operatorname{diam}(G)+1
\end{gathered}
$$

2.3 THEOREM : Let $I=\left\{i_{1}, i_{2}, \ldots ., i_{n}\right\}$ be an interval family and $G$ is an interval graph with $\delta(G) \geq 2$ corresponding to $I$. If $i, j, k$ are three consecutive intervals such that $i<j<k$ and $j$ is dominated interval, $i$ intersect $j, j$ intersect $k$ and $i$ intersect $k$. Then $\operatorname{rc}(G) \leq \operatorname{diam}(G)+1$.

PROOF : Let $I=\left\{i_{1}, i_{2}, \ldots ., i_{n}\right\}$ be an interval family and $G$ is an interval graph with $\delta(G) \geq 2$ of $I$. Let $i, j, k$ be three consecutive intervals satisfying the hypothesis of $G$, that is $\delta(G) \geq 2$. Now $i$

and $k$ intersect implies that $i$ and $k$ are adjacent in $G$, so that there will not any disconnection in $G$.

The graph $G$ must be $\delta(G) \geq 2$, where $\delta(G)$ is the minimum degree. The procedure as in Theorem 1, next we will prove that $\operatorname{rc}(G) \leq \operatorname{diam}(G)+1$ by using the following illustration.

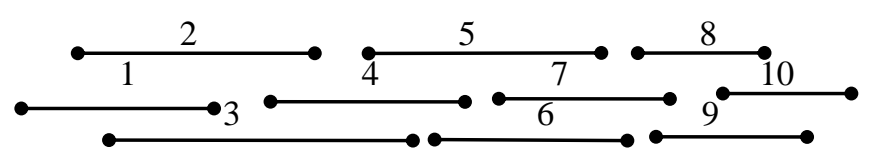

Fig.3: Interval family $I$

The Interval graph $G$ as follows from Interval family $I$ as in Fig.3 
$\operatorname{nbd}[1]=\{1,2,3\}$, nbd [2] $=\{1,2,3,4\}$,

$\operatorname{nbd}[5]=\{3,4,5,6,7\}, \operatorname{nbd}[6]=\{4,5,6,7,8\}$,

$\operatorname{nbd}[9]=\{7,8,9,10\}, \operatorname{nbd}[10]=\{8,9,10\}$ $\operatorname{nbd}[3]=\{1,2,3,4,5\}, \operatorname{nbd}[4]=\{2,3,4,5,6\}$, $\operatorname{nbd}[7]=\{5,6,7,8,9\}, \operatorname{nbd}[8]=\{6,7,8,9,10\}$,

The rainbow edge colors as follows from an Interval graph $\mathrm{G}$ corresponding to an Interval family I,

$\mathrm{C}\left(\mathrm{v}_{1}, \mathrm{v}_{2}\right)=1, \mathrm{C}\left(\mathrm{v}_{1}, \mathrm{v}_{3}\right)=2, \mathrm{C}\left(\mathrm{v}_{2}, \mathrm{v}_{3}\right)=3, \mathrm{C}\left(\mathrm{v}_{2}, \mathrm{v}_{4}\right)=2$,

$\mathrm{C}\left(\mathrm{v}_{3}, \mathrm{v}_{4}\right)=1, \mathrm{C}\left(\mathrm{v}_{3}, \mathrm{v}_{5}\right)=4, \mathrm{C}\left(\mathrm{v}_{4}, \mathrm{v}_{5}\right)=3, \mathrm{C}\left(\mathrm{v}_{4}, \mathrm{v}_{6}\right)=5$,

$\mathrm{C}\left(\mathrm{v}_{5}, \mathrm{v}_{6}\right)=1, \mathrm{C}\left(\mathrm{v}_{5}, \mathrm{v}_{7}\right)=2, \mathrm{C}\left(\mathrm{v}_{6}, \mathrm{v}_{7}\right)=3, \mathrm{C}\left(\mathrm{v}_{6}, \mathrm{v}_{8}\right)=3$,

$\mathrm{C}\left(\mathrm{v}_{7}, \mathrm{v}_{8}\right)=1, \mathrm{C}\left(\mathrm{v}_{7}, \mathrm{v}_{9}\right)=5, \mathrm{C}\left(\mathrm{v}_{8}, \mathrm{v}_{9}\right)=2, \mathrm{C}\left(\mathrm{v}_{8}, \mathrm{v}_{10}\right)=4$,

$\mathrm{C}\left(\mathrm{v}_{9}, \mathrm{v}_{10}\right)=1$

Therefore the set of colors are

$$
\begin{array}{r}
\mathrm{C}=\{1,2,3,2,1,4,3,5,1,2,3,3,1,5,2,4,1\} \\
=\{1,2,3,4,5\} \\
\therefore \operatorname{rc}(G)=5
\end{array}
$$

\begin{tabular}{|c|c|c|c|c|}
\hline $\mathrm{d}\left(\mathrm{v}_{1}, \mathrm{v}_{\mathrm{l}}\right.$ & $\left(v_{2}, v_{1}\right)=1$ & $1\left(v_{3}, v_{1}\right)=1$ & $(1)=2$ & $d\left(v_{5}\right.$ \\
\hline S & $\left(v_{2}, v_{2}\right)=0$ & $\left(v_{3}, v_{2}\right)=$ & $=$ & $d\left(v_{5}\right.$ \\
\hline$\left(\mathrm{v}_{1}, \mathrm{v}_{2}\right)$ & $\left(v_{2}, v_{3}\right)=1$ & 1 & 1 & $=2$ \\
\hline 1 & $\left(\mathrm{v}_{2}, \mathrm{v}_{4}\right)=1$ & $\mathrm{~d}\left(\mathrm{v}_{3}, \mathrm{v}_{3}\right)=$ & $\mathrm{d}\left(\mathrm{v}_{4}, \mathrm{v}_{3}\right)=$ & $d\left(v_{5}, v_{3}\right)$ \\
\hline$\left(\mathrm{v}_{1}, \mathrm{v}_{3}\right)$ & $\left(v_{2}, v_{5}\right)=2$ & 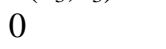 & 1 & $=1$ \\
\hline 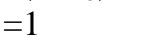 & $=2$ & 4) $=$ & $\left(v_{4}, v_{4}\right)=$ & $\mathrm{d}\left(\mathrm{v}_{5}, \mathrm{v}_{4}\right)=$ \\
\hline$\left(\mathrm{v}_{1}, \mathrm{v}_{4}\right)$ & $\left(v_{2}, v_{7}\right)=3$ & 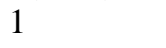 & & 1 \\
\hline 2 & $=3$ & )$=$ & )$=$ & $\mathrm{d}\left(\mathrm{v}_{5}\right.$, \\
\hline$\left(\mathrm{v}_{1}, \mathrm{v}_{5}\right)$ & & & & 0 \\
\hline$=2$ & $\left(\mathrm{v}_{2}, \mathrm{v}_{10}\right)=$ & $d\left(v_{3}, v_{6}\right)=$ & $\mathrm{d}\left(\mathrm{v}_{4}, \mathrm{v}_{6}\right)=$ & $\mathrm{d}\left(\mathrm{v}_{5}, \mathrm{v}_{6}\right)=$ \\
\hline$\left(\mathrm{v}_{1}, \mathrm{v}_{6}\right)$ & & & 1 & 1 \\
\hline$=3$ & $\begin{array}{l}\mathrm{d}\left(\mathrm{v}_{7}, \mathrm{v}_{1}\right)= \\
3\end{array}$ & $\mathrm{~d}\left(\mathrm{v}_{3}, \mathrm{v}_{7}\right)=$ & $\mathrm{d}\left(\mathrm{v}_{4}, \mathrm{v}_{7}\right)=$ & $\begin{array}{l}\mathrm{d}\left(\mathrm{v}_{5}, \mathrm{v}_{7}\right)= \\
1\end{array}$ \\
\hline 3 & $\begin{array}{l}\mathrm{d}\left(\mathrm{v}_{7}, \mathrm{v}_{2}\right)= \\
3\end{array}$ & $\mathrm{~d}\left(\mathrm{v}_{3}, \mathrm{v}_{8}\right)=$ & $\left(\mathrm{v}_{4}, \mathrm{v}_{8}\right)=$ & $8)=$ \\
\hline$=4$ & $\mathrm{~d}\left(\mathrm{v}_{7}, \mathrm{v}_{3}\right)=$ & $=$ & )$=$ & $\left(v_{5}, v_{9}\right)=$ \\
\hline $\mathrm{d}\left(\mathrm{v}_{1}, \mathrm{v}_{9}\right)$ & & & & \\
\hline$=4$ & $\mathrm{~d}\left(\mathrm{v}_{7}, \mathrm{v}_{4}\right)=$ & $d\left(v_{3}, v_{10}\right)=$ & $\left.v_{10}\right)$ & $\mathrm{d}\left(\mathrm{v}_{5}, \mathrm{v}_{10}\right)=$ \\
\hline $\mathrm{d}\left(\mathrm{v}_{1}, \mathrm{v}_{10}\right.$ & & & & \\
\hline & $\mathrm{d}\left(\mathrm{v}_{7}, \mathrm{v}_{5}\right)=$ & $\mathrm{d}\left(\mathrm{v}_{8}, \mathrm{v}_{1}\right)=$ & $\mathrm{d}\left(\mathrm{v}_{9}, \mathrm{v}_{1}\right)=$ & $\mathrm{d}\left(\mathrm{v}_{10}, \mathrm{v}_{1}\right)=$ \\
\hline $\mathrm{d}\left(\mathrm{v}_{6}, \mathrm{v}_{1}\right)=$ & & & & $d(v-v)=$ \\
\hline $\begin{array}{l}3 \\
d\left(v_{t}\right.\end{array}$ & $\begin{array}{l}\mathrm{d}\left(\mathrm{v}_{7}, \mathrm{v}_{6}\right)= \\
1\end{array}$ & $\begin{array}{l}\mathrm{d}\left(\mathrm{v}_{8}, \mathrm{v}_{2}\right)= \\
3\end{array}$ & $\mathrm{~d}\left(\mathrm{v}_{9}, \mathrm{v}_{2}\right)=$ & $\begin{array}{l}\mathrm{d}\left(\mathrm{v}_{10}, \mathrm{v}_{2}\right)= \\
4\end{array}$ \\
\hline $\begin{array}{l}2 \\
d\left(v_{6}, v_{3}\right)=\end{array}$ & $\begin{array}{l}\mathrm{d}\left(\mathrm{v}_{7}, \mathrm{v}_{7}\right)= \\
0\end{array}$ & $\mathrm{~d}\left(\mathrm{v}_{8}, \mathrm{v}_{3}\right)=$ & $\mathrm{d}\left(\mathrm{v}_{9}, \mathrm{v}_{3}\right)=$ & $\left(\mathrm{v}_{10}, \mathrm{v}_{3}\right)=$ \\
\hline $\begin{array}{l}2 \\
d(v\end{array}$ & $\begin{array}{l}\mathrm{d}\left(\mathrm{v}_{7}, \mathrm{v}_{8}\right)= \\
1\end{array}$ & $\mathrm{~d}\left(\mathrm{v}_{8}, \mathrm{v}_{4}\right)=$ & $\begin{array}{l}\mathrm{d}\left(\mathrm{v}_{9}, \mathrm{v}_{4}\right)= \\
3\end{array}$ & $\begin{array}{l}\mathrm{d}\left(\mathrm{v}_{10}, \mathrm{v}_{4}\right)= \\
3\end{array}$ \\
\hline 1 & $\mathrm{~d}\left(\mathrm{v}_{7}, \mathrm{v}_{9}\right)=$ & $\left.I_{5}\right)=$ & $d\left(v_{9}, v_{5}\right)=$ & $\mathrm{d}\left(\mathrm{v}_{10}, \mathrm{v}_{5}\right)=$ \\
\hline $\mathrm{d}\left(\mathrm{v}_{6}, \mathrm{v}_{5}\right)=$ & & & & \\
\hline $\mathrm{d}\left(\mathrm{v}_{6}, \mathrm{v}_{6}\right)$ & $\begin{array}{l}\mathrm{d}\left(\mathrm{v}_{7}, \mathrm{v}_{10}\right) \\
=2\end{array}$ & $\begin{array}{l}\mathrm{d}\left(\mathrm{v}_{8},\right. \\
1\end{array}$ & $\mathrm{~d}\left(\mathrm{v}_{9}, \mathrm{v}_{6}\right)$ & $\mathrm{d}\left(\mathrm{v}_{10}, \mathrm{v}_{6}\right)=$ \\
\hline $\begin{array}{l}=0 \\
\mathrm{~d}\left(\mathrm{v}_{6}, \mathrm{v}_{7}\right)\end{array}$ & & $\begin{array}{l}\mathrm{d}\left(\mathrm{v}_{8}, \mathrm{v}_{7}\right)= \\
1\end{array}$ & $\begin{array}{l}\mathrm{d}\left(\mathrm{v}_{9}, \mathrm{v}_{7}\right)= \\
1\end{array}$ & $\mathrm{~d}\left(\mathrm{v}_{10}, \mathrm{v}_{7}\right)=$ \\
\hline$=1$ & & $=$ & $\mathrm{d}(\mathrm{v}$ & $\mathrm{d}\left(\mathrm{v}_{10}, \mathrm{v}_{8}\right)=$ \\
\hline $\mathrm{d}\left(\mathrm{v}_{6}, \mathrm{v}_{8}\right)$ & & & & 2 \\
\hline$=1$ & & $\mathrm{~d}\left(\mathrm{v}_{8}, \mathrm{v}_{9}\right)=$ & $\mathrm{d}\left(\mathrm{v}_{9}, \mathrm{v}_{9}\right)=$ & $\mathrm{d}\left(\mathrm{v}_{10}, \mathrm{v}_{9}\right)=$ \\
\hline$=2$ & & $\mathrm{~d}\left(\mathrm{v}_{8}, \mathrm{v}_{10}\right)$ & $\mathrm{d}\left(\mathrm{v}_{9}, \mathrm{v}_{10}\right)=$ & $\mathrm{d}\left(\mathrm{v}_{10}, \mathrm{v}_{10}\right)=$ \\
\hline
\end{tabular}

The distances of the path lengths from an Interval graph $G$ as follows, 


$\mathrm{d}\left(\mathrm{v}_{6}, \mathrm{v}_{10}\right)=\quad=1 \quad 1 \quad 0$

2

The eccentricity of the vertices is as follows,

$$
\operatorname{ecc}(v)=\max _{x \in V(G)} d(v, x)
$$

$\operatorname{ecc}\left(\mathrm{v}_{1}\right)=\max \left\{\mathrm{d}\left(\mathrm{v}_{1}, \mathrm{v}_{1}\right), \mathrm{d}\left(\mathrm{v}_{1}, \mathrm{v}_{2}\right), \mathrm{d}\left(\mathrm{v}_{1}, \mathrm{v}_{3}\right), \mathrm{d}\left(\mathrm{v}_{1}, \mathrm{v}_{4}\right), \mathrm{d}\left(\mathrm{v}_{1}, \mathrm{v}_{5}\right), \mathrm{d}\left(\mathrm{v}_{1}, \mathrm{v}_{6}\right), \mathrm{d}\left(\mathrm{v}_{1}, \mathrm{v}_{7}\right), \mathrm{d}\left(\mathrm{v}_{1}, \mathrm{v}_{8}\right), \mathrm{d}\left(\mathrm{v}_{1}, \mathrm{v}_{9}\right), \mathrm{d}\left(\mathrm{v}_{1}, \mathrm{v}_{10}\right)\right\}$ $=\max \{0,1,1,2,2,3,3,4,4,5\}=5$

$\operatorname{ecc}\left(\mathrm{v}_{2}\right)=\max \left\{\mathrm{d}\left(\mathrm{v}_{2}, \mathrm{v}_{1}\right), \mathrm{d}\left(\mathrm{v}_{2}, \mathrm{v}_{2}\right), \mathrm{d}\left(\mathrm{v}_{2}, \mathrm{v}_{3}\right), \mathrm{d}\left(\mathrm{v}_{2}, \mathrm{v}_{4}\right), \mathrm{d}\left(\mathrm{v}_{2}, \mathrm{v}_{5}\right), \mathrm{d}\left(\mathrm{v}_{2}, \mathrm{v}_{6}\right), \mathrm{d}\left(\mathrm{v}_{2}, \mathrm{v}_{7}\right), \mathrm{d}\left(\mathrm{v}_{2}, \mathrm{v}_{8}\right), \mathrm{d}\left(\mathrm{v}_{2}, \mathrm{v}_{9}\right), \mathrm{d}\left(\mathrm{v}_{2}, \mathrm{v}_{10}\right)\right\}$ $=\max \{1,0,1,1,2,2,3,3,4,4\}=4$

$\operatorname{ecc}\left(\mathrm{v}_{3}\right)=\max \left\{d\left(\mathrm{v}_{3}, \mathrm{v}_{1}\right), \mathrm{d}\left(\mathrm{v}_{3}, \mathrm{v}_{2}\right), \mathrm{d}\left(\mathrm{v}_{3}, \mathrm{v}_{3}\right), \mathrm{d}\left(\mathrm{v}_{3}, \mathrm{v}_{4}\right), \mathrm{d}\left(\mathrm{v}_{3}, \mathrm{v}_{5}\right), \mathrm{d}\left(\mathrm{v}_{3}, \mathrm{v}_{6}\right), \mathrm{d}\left(\mathrm{v}_{3}, \mathrm{v}_{7}\right), \mathrm{d}\left(\mathrm{v}_{3}, \mathrm{v}_{8}\right), \mathrm{d}\left(\mathrm{v}_{3}, \mathrm{v}_{9}\right), \mathrm{d}\left(\mathrm{v}_{3}, \mathrm{v}_{10}\right)\right\}$ $=\max \{1,1,0,1,1,2,2,3,3,4\}=4$

$\operatorname{ecc}\left(\mathrm{v}_{4}\right)=\max \left\{\mathrm{d}\left(\mathrm{v}_{4}, \mathrm{v}_{1}\right), \mathrm{d}\left(\mathrm{v}_{4}, \mathrm{v}_{2}\right), \mathrm{d}\left(\mathrm{v}_{4}, \mathrm{v}_{3}\right), \mathrm{d}\left(\mathrm{v}_{4}, \mathrm{v}_{4}\right), \mathrm{d}\left(\mathrm{v}_{4}, \mathrm{v}_{5}\right), \mathrm{d}\left(\mathrm{v}_{4}, \mathrm{v}_{6}\right), \mathrm{d}\left(\mathrm{v}_{4}, \mathrm{v}_{7}\right), \mathrm{d}\left(\mathrm{v}_{4}, \mathrm{v}_{8}\right), \mathrm{d}\left(\mathrm{v}_{4}, \mathrm{v}_{9}\right), \mathrm{d}\left(\mathrm{v}_{4}, \mathrm{v}_{10}\right)\right\}$ $=\max \{2,1,1,0,1,1,2,2,3,3\}=3$

$\operatorname{ecc}\left(\mathrm{v}_{5}\right)=\max \left\{\mathrm{d}\left(\mathrm{v}_{5}, \mathrm{v}_{1}\right), \mathrm{d}\left(\mathrm{v}_{5}, \mathrm{v}_{2}\right), \mathrm{d}\left(\mathrm{v}_{5}, \mathrm{v}_{3}\right), \mathrm{d}\left(\mathrm{v}_{5}, \mathrm{v}_{4}\right), \mathrm{d}\left(\mathrm{v}_{5}, \mathrm{v}_{5}\right), \mathrm{d}\left(\mathrm{v}_{5}, \mathrm{v}_{6}\right), \mathrm{d}\left(\mathrm{v}_{5}, \mathrm{v}_{7}\right), \mathrm{d}\left(\mathrm{v}_{5}, \mathrm{v}_{8}\right), \mathrm{d}\left(\mathrm{v}_{5}, \mathrm{v}_{9}\right), \mathrm{d}\left(\mathrm{v}_{5}, \mathrm{v}_{10}\right)\right\}$ $=\max \{2,2,1,1,0,1,1,2,2,3\}=3$

$\operatorname{ecc}\left(\mathrm{v}_{6}\right)=\max \left\{\mathrm{d}\left(\mathrm{v}_{6}, \mathrm{v}_{1}\right), \mathrm{d}\left(\mathrm{v}_{6}, \mathrm{v}_{2}\right), \mathrm{d}\left(\mathrm{v}_{6}, \mathrm{v}_{3}\right), \mathrm{d}\left(\mathrm{v}_{6}, \mathrm{v}_{4}\right), \mathrm{d}\left(\mathrm{v}_{6}, \mathrm{v}_{5}\right), \mathrm{d}\left(\mathrm{v}_{6}, \mathrm{v}_{6}\right), \mathrm{d}\left(\mathrm{v}_{6}, \mathrm{v}_{7}\right), \mathrm{d}\left(\mathrm{v}_{6}, \mathrm{v}_{8}\right), \mathrm{d}\left(\mathrm{v}_{6}, \mathrm{v}_{9}\right), \mathrm{d}\left(\mathrm{v}_{6}, \mathrm{v}_{10}\right)\right\}$ $=\max \{3,2,2,1,1,0,1,1,2,2\}=3$

$\operatorname{ecc}\left(\mathrm{v}_{7}\right)=\max \left\{\mathrm{d}\left(\mathrm{v}_{7}, \mathrm{v}_{1}\right), \mathrm{d}\left(\mathrm{v}_{7}, \mathrm{v}_{2}\right), \mathrm{d}\left(\mathrm{v}_{7}, \mathrm{v}_{3}\right), \mathrm{d}\left(\mathrm{v}_{7}, \mathrm{v}_{4}\right), \mathrm{d}\left(\mathrm{v}_{7}, \mathrm{v}_{5}\right), \mathrm{d}\left(\mathrm{v}_{7}, \mathrm{v}_{6}\right), \mathrm{d}\left(\mathrm{v}_{7}, \mathrm{v}_{7}\right), \mathrm{d}\left(\mathrm{v}_{7}, \mathrm{v}_{8}\right), \mathrm{d}\left(\mathrm{v}_{7}, \mathrm{v}_{9}\right), \mathrm{d}\left(\mathrm{v}_{7}, \mathrm{v}_{10}\right)\right\}$ $=\max \{3,3,2,2,1,1,0,1,1,2\}=3$

$\operatorname{ecc}\left(\mathrm{v}_{8}\right)=\max \left\{\mathrm{d}\left(\mathrm{v}_{8}, \mathrm{v}_{1}\right), \mathrm{d}\left(\mathrm{v}_{8}, \mathrm{v}_{2}\right), \mathrm{d}\left(\mathrm{v}_{8}, \mathrm{v}_{3}\right), \mathrm{d}\left(\mathrm{v}_{8}, \mathrm{v}_{4}\right), \mathrm{d}\left(\mathrm{v}_{8}, \mathrm{v}_{5}\right), \mathrm{d}\left(\mathrm{v}_{8}, \mathrm{v}_{6}\right), \mathrm{d}\left(\mathrm{v}_{8}, \mathrm{v}_{7}\right), \mathrm{d}\left(\mathrm{v}_{8}, \mathrm{v}_{8}\right), \mathrm{d}\left(\mathrm{v}_{8}, \mathrm{v}_{9}\right), \mathrm{d}\left(\mathrm{v}_{8}, \mathrm{v}_{10}\right)\right\}$ $=\max \{4,3,3,2,2,1,1,0,1,1\}=4$

$\operatorname{ecc}\left(\mathrm{v}_{9}\right)=\max \left\{\mathrm{d}\left(\mathrm{v}_{9}, \mathrm{v}_{1}\right), \mathrm{d}\left(\mathrm{v}_{9}, \mathrm{v}_{2}\right), \mathrm{d}\left(\mathrm{v}_{9}, \mathrm{v}_{3}\right), \mathrm{d}\left(\mathrm{v}_{9}, \mathrm{v}_{4}\right), \mathrm{d}\left(\mathrm{v}_{9}, \mathrm{v}_{5}\right), \mathrm{d}\left(\mathrm{v}_{9}, \mathrm{v}_{6}\right), \mathrm{d}\left(\mathrm{v}_{9}, \mathrm{v}_{7}\right), \mathrm{d}\left(\mathrm{v}_{9}, \mathrm{v}_{8}\right), \mathrm{d}\left(\mathrm{v}_{9}, \mathrm{v}_{9}\right), \mathrm{d}\left(\mathrm{v}_{9}, \mathrm{v}_{10}\right)\right\}$ $=\max \{4,4,3,3,2,2,1,1,0,1\}=4$

$\operatorname{ecc}\left(\mathrm{v}_{10}\right)=\max \left\{\mathrm{d}\left(\mathrm{v}_{10}, \mathrm{v}_{1}\right), \mathrm{d}\left(\mathrm{v}_{10}, \mathrm{v}_{2}\right), \mathrm{d}\left(\mathrm{v}_{10}, \mathrm{v}_{3}\right), \mathrm{d}\left(\mathrm{v}_{10}, \mathrm{v}_{4}\right), \mathrm{d}\left(\mathrm{v}_{10}, \mathrm{v}_{5}\right), \mathrm{d}\left(\mathrm{v}_{10}, \mathrm{v}_{6}\right), \mathrm{d}\left(\mathrm{v}_{10}, \mathrm{v}_{7}\right), \mathrm{d}\left(\mathrm{v}_{10}, \mathrm{v}_{8}\right), \mathrm{d}\left(\mathrm{v}_{10}, \mathrm{v}_{9}\right)\right.$, $\left.\mathrm{d}\left(\mathrm{v}_{10}, \mathrm{v}_{10}\right)\right\}$ $=\max \{5,4,4,3,3,3,3,4,4,5\}=5$

The diameter of a graph $G$ is the maximum of eccentricity of all its vertices and is denoted by $\operatorname{diam}(G)$.

$$
\begin{aligned}
& \operatorname{diam}(G)=\max \left\{\operatorname{ecc}\left(v_{1}\right), \operatorname{ecc}\left(v_{2}\right), \operatorname{ecc}\left(v_{3}\right),\right. \\
& \operatorname{ecc}\left(v_{4}\right), \operatorname{ecc}\left(v_{5}\right), \operatorname{ecc}\left(v_{6}\right), \operatorname{ecc}\left(v_{7}\right), \operatorname{ecc}\left(v_{8}\right), \\
& \left.\operatorname{ecc}\left(v_{9}\right), \operatorname{ecc}\left(v_{10}\right)\right\} \\
& =\max \{5,4,4,3,3,3,3,4,4,5\}=5 \\
& \therefore \operatorname{rc}(\mathrm{G})=5, \operatorname{diam}(\mathrm{G})+1=6 \\
& \therefore \operatorname{rc}(G) \leq \operatorname{diam}(G)+1
\end{aligned}
$$

\section{CONCLUSION}

The interval graphs are rich in combinatorial structure and have found applications in several disciplines such as Tropic control, Ecology, Biology and Computer Science and particularly useful in real line scheduling and computer storage allocation problems. In this paper we individualized an interval graphs as various digraphs. We then extended the results to trace out a specific type of an interval graphs having every pair of vertices as a minimum rainbow coloring. Moreover we presented that every vertex of an interval graph has the minimum degree two. 


\section{REFERENCES}

[1] Y. Caro, A. Lev, Y. Roditty, Z. Tuza, and R. Yuster, On rainbow connection, Electr J Combin 15(R57) (2008), 1.

[2] G. Chartrand, G.L. Johns,K.A. McKeon, and P. Zhang, Rainbow connection in graphs, Math Bohemica 133(1) (2008), 5-98.

[3] P. Erdos, J. Pach, R. Pollack, and Z. Tuza, Radius, diameter and minimum degree, J Combin Theory, Ser B 47(1) (1989), 73-79.

[4] I. Schiermeyer, Rainbow connection in graphs with minimum degree three, in : Combinatorial Algorithms, Lecture Notes in Computer Science 5874 (J. Fiala, J. Kratochvl, and M. Miller, Eds.), Springer Berlin/ Heidelberg, 2009, pp. $432-437$.

[5] S. Chakraborty, E. Fischer, A. Matsliah, and R. Yuster, Hardness and algorithms for rainbow connection, J Combin Optimiz (2009), $1-18$. 